\title{
Structural and magnetic investigations of post-perovskite thiocyanate frameworks
}

\author{
M. Geers ${ }^{1,2}$, L. Cañadillas Delgado², O. Fabelo², M. Cliffe ${ }^{1}$ \\ ${ }^{1}$ School of Chemistry, University of Nottingham, University Park, Nottingham, NG7 2RD, UK, \\ ${ }^{2}$ Institut Laue Langevin, 71 Avenue des Martyrs, CS 20156, 38042 Grenoble Cedex 9, FRANCE \\ geers@ill.fr
}

Dense coordination polymers combine the functional properties typical of the traditional inorganic solid state, such as magnetism, with the remarkable tunability and flexibility that arises from the incorporation of molecular components. They therefore offer the opportunity to discover unusual behaviour that arises from the coupling of these properties. [1] Thiocyanate compounds have the potential for rich optical and magnetic properties, but both their chemistry and magnetism remain comparatively unexplored.

Metal framework compounds involving bridging thiocyanate ligands have started to establish themselves as a rewarding family of materials for magnetic studies, as the thiocyanate acts as an effective superexchange pathway between metal centres. [2] In addition, the asymmetric bonding requirements of the nitrogen and sulphur termini can often result in unconventional framework topologies. This, in turn, can lead to unusual magnetic structures and spin textures in the compounds.

Here will be presented three isomorphous metal thiocyanate frameworks, $\operatorname{CsM}(\mathrm{NCS})_{3}$, where $\mathrm{M}=\mathrm{Ni}$, [3] $\mathrm{Mn}$ and $\mathrm{Co}$; of which the latter two are new materials. The structures of these materials have been determined by single crystal X-ray diffraction to be postperovskite two-dimensional frameworks with interplanar Cs counterions. Bulk magnetic susceptibility measurements revealed all the materials magnetically order between 6 and $16 \mathrm{~K}$, with complex, non-collinear orderings. Following these results, neutron diffraction experiments of both single crystal and powder samples have been carried out to explore the unusual magnetic features further.

[1] W. Li, et al., Nat. Rev. Mater., 2017, 2, 16099.

[2] E. Bassey, et al., Inorg. Chem., 2020, 59, 11627 - 11639.

[3] M. Fleck, Acta Crystallogr. C60, i63 (2004).

Keywords: metal thiocyanate framework; molecular magnetism; neutron diffraction 